
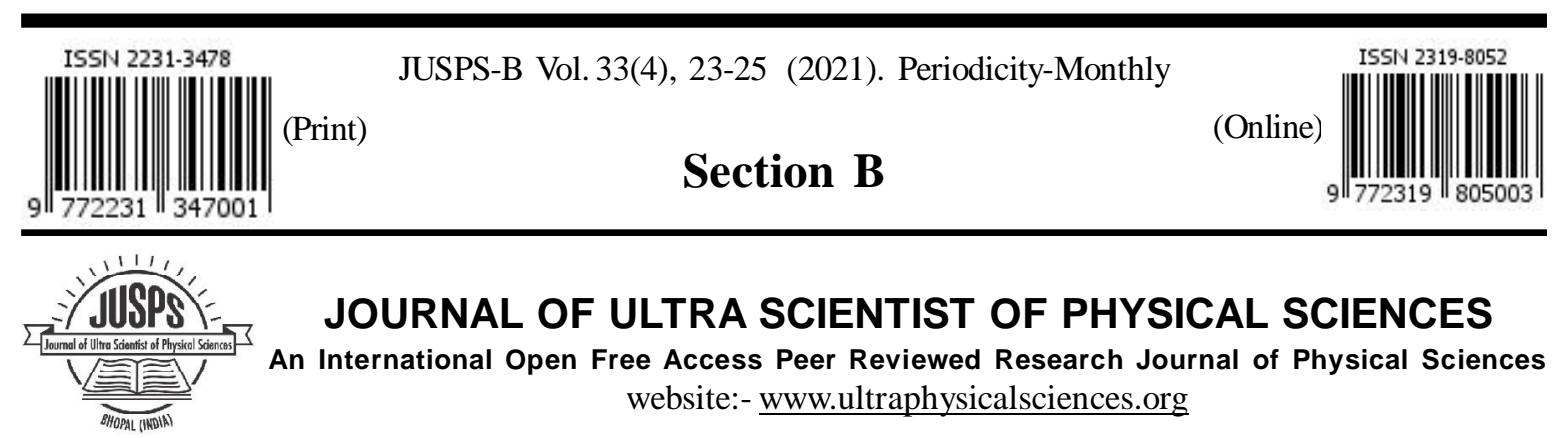

JOURNAL OF ULTRA SCIENTIST OF PHYSICAL SCIENCES

An International Open Free Access Peer Reviewed Research Journal of Physical Sciences website:- www.ultraphysicalsciences.org

Estd. 1989

\title{
A new Type of Fixed Point Theorem in a Complete Dislocated Metric Space
}

\author{
YASHVIR SINGH \\ P. G. Department and Research Centre of Mathematics, Hindu College \\ Moradabad, U.P. (India) \\ Corresponding Author E.mail : yvs170901@ gmail.com \\ http://dx.doi.org/10.22147/jusps-B/330402
}

Acceptance Date 10th September, 2021, Online Publication Date 26th September, 2021

\begin{abstract}
In this paper a fixed point theorem have been proved in dislocated metric spaces using a class of continuous function $\mathrm{G}_{4}$.

Key words: fixed point, dislocated metric space.

AMS Subject Classification: 47H10, 54H25.

\section{Introduction}

The Polish mathematician Stefan Banach in 1922 proved a theorem which ensures, under appropriate conditions, the existence and uniqueness of a fixed point. It is well known as a Banach fixed point theorem. The existence of a fixed point plays an important role in several areas of mathematics, physics and engineering branches. This principle has been generalized by many authors in various ways. Hitzler and Seda ${ }^{8}$ introduced the concept of dislocated metric space, which is generalized by Zeyada et al. ${ }^{3}$ by introducing the concept of dislocated quasi-metric space. The aim of this paper is to obtain a fixed point theorem in dislocated metric space. Our result generalizes the main result of Kastriot and Elida ${ }^{7}$.
\end{abstract}

\section{Preliminaries:} definitions

Definition $^{3}$ : Let $\mathrm{X}$ be a non empty set and let $\mathrm{d}: \mathrm{X} \times \mathrm{X} \rightarrow[0, \infty)$ be a function satisfying following (i) $\quad \mathrm{d}(\mathrm{x}, \mathrm{y})=\mathrm{d}(\mathrm{y}, \mathrm{x})=0$ implies $\mathrm{x}=\mathrm{y}$.

This is an open access article under the CC BY-NC-SA license (https://creativecommons.org/licenses/by-nc-sa/4.0) 
(ii) $\mathrm{d}(\mathrm{x}, \mathrm{y}) \leq \mathrm{d}(\mathrm{x}, \mathrm{z})+\mathrm{d}(\mathrm{z}, \mathrm{y})$ for all $\mathrm{x}, \mathrm{y}, \mathrm{z} \in \mathrm{X}$.

Then $\mathrm{d}$ is called a dislocated quasi-metric on $\mathrm{X}$. If $\mathrm{d}$ satisfies $\mathrm{d}(\mathrm{x}, \mathrm{y})=0$, then it is called a quasi-metric on $X$. If $d$ satisfies $d(x, y)=d(y, x)$, then it is called dislocated metric.

Definition $^{3}$ : A sequence $\left\{\mathrm{x}_{\mathrm{n}}\right\}$ in dq-metric space (dislocated quasi-metric space) (X, d) is called Cauchy sequence if for, given $\varepsilon>0$, there exist $\mathrm{n}_{0} \in \mathrm{N}$, such that $\forall \mathrm{m}, \mathrm{n} \geq \mathrm{n}_{0}$, implies $\mathrm{d}\left(\mathrm{x}_{\mathrm{m}}, \mathrm{x}_{\mathrm{n}}\right)<\varepsilon$ or $\mathrm{d}\left(\mathrm{x}_{\mathrm{n}}, \mathrm{x}_{\mathrm{m}}\right)<\varepsilon$ i.e. $\min \left\{\mathrm{d}\left(\mathrm{x}_{\mathrm{m}}, \mathrm{x}_{\mathrm{n}}\right), \mathrm{d}\left(\mathrm{x}_{\mathrm{n}}, \mathrm{x}_{\mathrm{m}}\right)\right\}<\varepsilon$.

Definition $^{3}$ : A sequence $\left\{\mathrm{x}_{\mathrm{n}}\right\}$ dislocated quasi-convergent to $\mathrm{x}$ if $\lim _{n \rightarrow \infty} d\left(x_{n}, x\right)=\lim _{n \rightarrow \infty} d\left(x, x_{n}\right)=0$

In this case $\mathrm{x}$ is called a dq-limit of $\left\{\mathrm{x}_{\mathrm{n}}\right\}$ and we write $\mathrm{x}_{\mathrm{n}} \rightarrow \mathrm{x}$.

Definition $^{3}$ : A dq-metric space $(\mathrm{X}, \mathrm{d})$ is called complete if every Cauchy sequence in it is a dqconvergent.

Definition $^{4}$ : Let $(\mathrm{X}, \mathrm{d})$ be a dq-metric space. A map $\mathrm{T}: \mathrm{X} \rightarrow \mathrm{X}$ is called contraction if there exists $0 \leq \lambda$ $\leq 1$ such that

$$
\mathrm{d}(\mathrm{Tx}, \mathrm{Ty}) \leq \lambda \mathrm{d}(\mathrm{x}, \mathrm{y}) \text {, for all } \mathrm{x}, \mathrm{y} \in \mathrm{X}
$$

\section{Main Result}

We consider the set $\mathrm{G}_{4}$ of all continuous functions $\mathrm{g}:[0, \infty)^{4} \rightarrow[0, \infty)$ with the following properties

(1) $\mathrm{g}$ is non decreasing in respect to each variable.

(2) $\mathrm{g}(\mathrm{t}, \mathrm{t}, \mathrm{t}, \mathrm{t}) \leq \mathrm{t}$ for all $\mathrm{t} \in[0, \infty)$.

For example $\mathrm{g}\left(\mathrm{t}_{1}, \mathrm{t}_{2}, \mathrm{t}_{3}, \mathrm{t}_{4}\right)=\max \left\{\mathrm{t}_{1}, \mathrm{t}_{2}, \mathrm{t}_{3}, \mathrm{t}_{4}\right\}$ or $\max \left\{\mathrm{t}_{1}, \mathrm{t}_{2}, \mathrm{t}_{2}, \mathrm{t}_{3}, \mathrm{t}_{3}+\mathrm{t}_{4}, \mathrm{t}_{4}+\mathrm{t}_{1}\right\}$.

Theorem : Let $(\mathrm{X}, \mathrm{d})$ be a complete dislocated metric space and $\mathrm{f}, \mathrm{g}: \mathrm{X} \rightarrow \mathrm{X}$ be two continuous mappings such that

$d(f x, g y) \leq \operatorname{cg}[d(x, y), d(x, f x), d(y, g y), d(f y, g x)]$

for all $\mathrm{x}, \mathrm{y} \in \mathrm{X}$ where $\mathrm{g} \in \mathrm{G}_{4}$ and $0 \leq \lambda<1$. Then $\mathrm{f}$ and $\mathrm{g}$ have a unique common fixed point in $\mathrm{X}$.

Proof: Let $\mathrm{x}_{\mathrm{o}} \in \mathrm{X}$ and define a sequence $\left\{\mathrm{x}_{\mathrm{n}}\right\}$ in $\mathrm{X}$ such that

$$
\begin{aligned}
& \mathrm{fx}_{0}=\mathrm{x}_{1}, \mathrm{gx}_{1}=\mathrm{x}_{2} \ldots \ldots \ldots, \mathrm{gx}_{2 \mathrm{n}-1}=\mathrm{x}_{2 \mathrm{n}}, \mathrm{fx}_{2 \mathrm{n}}=\mathrm{x}_{2 \mathrm{n}+1} \\
& \text { Consider, } \mathrm{d}\left(\mathrm{x}_{2 \mathrm{n}+1}, \mathrm{x}_{2 \mathrm{n}+2}\right)=\mathrm{d}\left(\mathrm{fx}_{2 \mathrm{n}}, \mathrm{gx}_{2 \mathrm{n}+1}\right) \\
& \leq \lambda \mathrm{g}\left[\mathrm{d}\left(\mathrm{x}_{2 \mathrm{n}}, \mathrm{x}_{2 \mathrm{n}+1}\right), \mathrm{d}\left(\mathrm{x}_{2 \mathrm{n}}, \mathrm{fx}_{2 \mathrm{n}}\right), \mathrm{d}\left(\mathrm{x}_{2 \mathrm{n}+1}, \mathrm{gx}_{2 \mathrm{n}+1}\right), \mathrm{d}\left(\mathrm{fx}_{2 \mathrm{n}+1}, \mathrm{gx}_{2 \mathrm{n}}\right)\right] \\
& \leq \lambda \mathrm{g}\left[\mathrm{d}\left(\mathrm{x}_{2 \mathrm{n}}, \mathrm{x}_{2 \mathrm{n}+1}\right), \mathrm{d}\left(\mathrm{x}_{2 \mathrm{n}}, \mathrm{fx}_{2 \mathrm{n}+1}\right), \mathrm{d}\left(\mathrm{x}_{2 \mathrm{n}+1}, \mathrm{x}_{2 \mathrm{n}+2}\right), \mathrm{d}\left(\mathrm{x}_{2 \mathrm{n}+2}, \mathrm{x}_{2 \mathrm{n}+2}\right)\right] \\
& \leq \lambda \mathrm{d}\left(\mathrm{x}_{2 \mathrm{n}}, \mathrm{x}_{2 \mathrm{n}+1}\right)
\end{aligned}
$$

So $d\left(x_{2 n+1}, x_{2 n+2}\right) \leq \lambda d\left(x_{2 n}, x_{2 n+1}\right)$

In a similar way we have, $d\left(x_{2 n}, x_{2 n+1}\right)=d\left(g x_{2 n-1}, f x_{2 n}\right)$

$$
\begin{aligned}
& \quad=d\left(\mathrm{fx}_{2 \mathrm{n}}, \mathrm{gx}_{2 \mathrm{n}-1}\right) \\
& \leq \lambda \mathrm{g}\left[\mathrm{d}\left(\mathrm{x}_{2 \mathrm{n}}, \mathrm{x}_{2 \mathrm{n}-1}\right), \mathrm{d}\left(\mathrm{x}_{2 \mathrm{n}}, \mathrm{fx} 2 \mathrm{n}\right), \mathrm{d}\left(\mathrm{x}_{2 \mathrm{n}-1}, \mathrm{gx}_{2 \mathrm{n}-1}\right), \mathrm{d}\left(\mathrm{fx}_{2 \mathrm{n}}, \mathrm{gx}_{2 \mathrm{n}-1}\right)\right] \\
& \leq \lambda \mathrm{g}\left[\mathrm{d}\left(\mathrm{x}_{2 \mathrm{n}}, \mathrm{x}_{2 \mathrm{n}-1}\right), \mathrm{d}\left(\mathrm{x}_{2 \mathrm{n}}, \mathrm{fx}_{2 \mathrm{n}+1}\right), \mathrm{d}\left(\mathrm{x}_{2 \mathrm{n}-1}, \mathrm{x}_{2 \mathrm{n}}\right), \mathrm{d}\left(\mathrm{x}_{2 \mathrm{n}}, \mathrm{x}_{2 \mathrm{n}+1}\right)\right] \\
& \leq \lambda \mathrm{d}\left(\mathrm{x}_{2 \mathrm{n}-1}, \mathrm{x}_{2 \mathrm{n}}\right)
\end{aligned}
$$


Continuing this process we get $\mathrm{d}\left(\mathrm{x}_{2 \mathrm{n}+1}, \mathrm{x}_{2 \mathrm{n}+2}\right) \leq \lambda \mathrm{d}\left(\mathrm{x}_{2 \mathrm{n}}, \mathrm{x}_{2 \mathrm{n}+1}\right) \leq$ $\leq \lambda^{2 \mathrm{n}} \mathrm{d}\left(\mathrm{x}_{0}, \mathrm{x}_{1}\right)$

Since $0 \leq \lambda<1$, as $\mathrm{n} \rightarrow \infty, \lambda^{\mathrm{n}} \rightarrow 0$ which implies that $\mathrm{d}\left(\mathrm{x}_{2 \mathrm{n}+1}, \mathrm{x}_{2 \mathrm{n}+2}\right) \rightarrow 0$. Hence $\left(\mathrm{x}_{\mathrm{n}}\right)$ is a cauchy sequence in complete dislocated metric space $X$. So by completeness of $X$ this sequence $\left(x_{n}\right)$ must be convergent to $u$ in $X$. Thus both the subsequence $\left(\mathrm{fx}_{2 \mathrm{n}}\right)$ and $\left(\mathrm{gX}_{2 \mathrm{n}}\right)$ also converges to $\mathrm{u}$. Since $\mathrm{f}, \mathrm{g}: \mathrm{X} \rightarrow \mathrm{X}$ are continuous mapping we get $\mathrm{fu}=\mathrm{u}$ and $\mathrm{gu}=\mathrm{u}$.

Uniqueness : Let $\mathrm{v} \neq \mathrm{u}$ be another fixed point of $\mathrm{f}$ and $\mathrm{g}$ where $\mathrm{fu}=\mathrm{u}$ and $\mathrm{gv}=\mathrm{v}$. Then by given condition, we have $\mathrm{d}(\mathrm{u}, \mathrm{v}) \leq \mathrm{d}(\mathrm{fu}, \mathrm{gv})$

$$
\begin{aligned}
& \leq \lambda \mathrm{g}[\mathrm{d}(\mathrm{u}, \mathrm{v}), \mathrm{d}(\mathrm{u}, \mathrm{fu}), \mathrm{d}(\mathrm{v}, \mathrm{gv}), \mathrm{d}(\mathrm{fv}, \mathrm{gu})] \\
& \leq \lambda \mathrm{g}[\mathrm{d}(\mathrm{u}, \mathrm{v}), \mathrm{d}(\mathrm{u}, \mathrm{u}), \mathrm{d}(\mathrm{v}, \mathrm{v}), \mathrm{d}(\mathrm{v}, \mathrm{u})]
\end{aligned}
$$

Replacing $v=u$ in $(2)$ we get, $d(u, u) \leq d(f u, g u)$

$$
\begin{aligned}
& \leq \lambda \mathrm{g}[\mathrm{d}(\mathrm{u}, \mathrm{u}), \mathrm{d}(\mathrm{u}, \mathrm{fu}), \mathrm{d}(\mathrm{u}, \mathrm{gu}), \mathrm{d}(\mathrm{fu}, \mathrm{gu})] \\
& \leq \lambda \mathrm{g}[\mathrm{d}(\mathrm{u}, \mathrm{u}), \mathrm{d}(\mathrm{u}, \mathrm{u}), \mathrm{d}(\mathrm{u}, \mathrm{u}), \mathrm{d}(\mathrm{u}, \mathrm{u})] \\
& \leq \lambda \mathrm{d}(\mathrm{u}, \mathrm{u})
\end{aligned}
$$

Thus we have $d(u, u)=0$, since $0 \leq \lambda B 1$.

Now replacing $\mathrm{u}=\mathrm{v}$ in $(2)$ we get $\mathrm{d}(\mathrm{v}, \mathrm{v})=0$.

Again from (2), we have

$$
d(u, v) d \text { " } \lambda d(u, v) \text { since } 0 \leq \lambda B 1 \text { we get } u=v \text {. }
$$

Thus fixed point is unique.

\section{References}

1. C.T. Aage, J.N. Salunke : The results on fixed points in dislocated and dislocated quasimetric space, Appl. Math. Sci., 2(59), 2941-2948 (2008).

2. B.E. Rhoadas : A comparison of various definitions of contractive mappings, Trans. Amer. Soc., 226, 257290 (1977).

3. F.M. Zeyada, G.H. Hassan, M.A. Ahmed : A generalization of fixed point theorem due to Hitzler and Seda in dislocated quasi-metric space, Areb. Jour. Sci. Eng., 31(1A), 111-114 (2005).

4. A. Isufati : Fixed point theorems in dislocated quasi-metric space, Appl. Math. Sci., 2(59), 2941-2948 (2008).

5. C.T. Aage, J.N. Salunke : Some results of fixed point theorem in dislocated quasi metric spaces, Bulletin of Marathwada Mathematical Society, 9, 1-9 (2008).

6. R. Srivastava, Z. Ansari, M. Sharma : Some results on fixed points in dislocated and dislocated quasi-metric spaces, Jour. Adv. Stud Topo., 3(1), 25-31 (2012).

7. Kastriot Zoto and Elida Hoxha : Fixed point theorems in dislocated and dislocated quasi-metric spaces, 3(4), 119-124 (2012).

8. P. Hitzler and A. K. Seda, Dislocated topologies, J. Electr. Eng., 51(12/s), 3-7.

9. P. Proinov : Fixed point theorems for generalized contractive mappings, J. Of Fixed Point Theory Appl., 22, 1-27 (2020).

10. O. Popescu : Some remarks on the proper "Fixed point theorems for generalized contractive mappings in metric spaces". J. Fixed Point Theory Appl., 23, 72 (2021). 